\title{
ESTUDO DA GERAÇÃO DE BOLHAS DE DIFERENTES TAMANHOS UTILIZANDO UM VENTURI EM COLUNA DE FLOTAÇÃO
}

\author{
A. M. R. FILHO ${ }^{1}$, L. L. CAMPOS ${ }^{1}$, V. J. S. MARQUES ${ }^{1}$, A. S. REIS ${ }^{1}$ e M. A. S. BARROZO ${ }^{1}$ \\ ${ }^{1}$ Universidade Federal de Uberlândia, Faculdade de Engenharia Química \\ E-mail para contato: sreis.angelica@gmail.com
}

\begin{abstract}
RESUMO - A técnica mais importante na concentração de minérios é a flotação, porém é um processo complexo no qual estão presentes fenômenos físicoquímicos e hidrodinâmicos. O processo de flotação baseia-se no conceito de que as partículas de valor (flotáveis) são capturadas pelas bolhas na fase líquida e carregadas até a interface polpa-espuma, enquanto que as partículas hidrofílicas são retidas no sistema. Sendo assim, o tamanho de bolha é uma das variáveis físicas mais importantes nesse processo, pois influencia diretamente na eficiência de captura das partículas. A utilização de tensoativos, no processo de flotação de partículas finas, tem como principal função controlar o tamanho de bolha, produzindo bolhas menores aumentando assim a probabilidade de colisão bolhapartícula. Desta forma, este trabalho teve por objetivo estudar a capacidade de um aerador tipo Venturi de gerar bolhas de diversos tamanhos, utilizando-se da adição de tensoativo e da variação da vazão de ar para promover os diferentes tamanhos de bolhas. Além disso, avaliar, também, a influência de partículas sólidas adicionadas ao sistema, no tamanho de bolhas. Os resultados obtidos permitiram observar as melhores condições de vazão de ar, concentração e vazão de tensoativo para geração de diferentes tamanhos de bolhas. Também foi possível verificar que adição de partículas ao sistema influi no tamanho das bolhas, reduzindo as mesmas significativamente.
\end{abstract}

\section{INTRODUÇÃO}

A flotação é um processo físico-químico bem complexo utilizado no beneficiamento de minérios, como a apatita, cujo objetivo é promover a separação das partículas de interesse dos minerais de ganga. Neste processo, bolhas de ar são introduzidas e/ou formadas no interior da coluna. Desse modo, as partículas hidrofóbicas se aderem à superfície das bolhas formando agregados bolha-partículas que ascendem (flotam), formando uma fase de espuma que é coletada no topo do equipamento.

Visto que o diâmetro de bolha é uma variável importante na otimização desse processo, a determinação e controle do tamanho das bolhas formadas tornam-se essenciais para caracterizar o processo de flotação e, assim, determinar os valores operacionais que resultem em melhores recuperações do mineral de interesse (Filho et al., 2015). 
Diversos estudos são realizados tendo como objetivo controlar o tamanho de bolha, sendo que, uma das formas mais eficazes, é através da adição de tensoativos. De forma geral, a estrutura molecular dos tensoativos (espumantes) apresenta uma parte hidrofóbica (apolar) e uma hidrofílica (polar). Comumente, a parte hidrofílica é representada pelo grupo $\mathrm{OH}$ (como no caso dos álcoois) e, a parte hidrofóbica é uma cadeia hidrocarbônica. Devido à sua bipolaridade, o espumante adsorve na superfície da bolha de maneira a ficar com a parte hidrofílica voltada para a água e a hidrofóbica para a bolha (Finch et al., 2008).

A grande maioria dos estudos e desenvolvimentos na flotação de partículas finas se baseia no aumento da probabilidade de colisão bolha-partícula. Sendo assim, a flotação para diversos tamanhos de partículas exige uma variação extensa da DTB (distribuição de tamanho de bolha). O tamanho das bolhas pode variar de 10-100 $\mu \mathrm{m}$ (microbolhas), 100-1000 $\mu \mathrm{m}$ (bolhas intermediárias) e 1000-4000 $\mu \mathrm{m}$ (bolhas grandes) (Reis e Barrozo, 2016) e estas podem ser obtidas utilizando-se aeradores externos como Venturi ou misturadores estáticos. A variação da vazão de ar e a adição de soluções tensoativas, por exemplo, podem controlar o tamanho das bolhas.

Diante do exposto, este trabalho teve como objetivo avaliar a capacidade de um aerador tipo Venturi de gerar bolhas de diversos tamanhos em uma coluna de flotação, utilizando-se da adição de tensoativo e da variação da vazão de ar. Além disso, verificou-se, também, a influência no tamanho de bolhas quando partículas sólidas são adicionadas ao sistema.

\section{MATERIAIS E MÉTODOS}

\subsection{Unidade Experimental}

A Figura 1 representa um esquema da unidade experimental a qual se encontra no Laboratório de Sistemas Particulados da Faculdade de Engenharia Química da Universidade Federal de Uberlândia.

A unidade consiste em uma coluna de flotação feita em acrílico dividida em três seções. Uma seção cilíndrica com $150 \mathrm{~cm}$ de comprimento e $4 \mathrm{~cm}$ de diâmetro, abaixo dessa a segunda seção, com formato tronco-cônica de $9,5 \mathrm{~cm}$ de altura e, logo após, a terceira seção de formato cilíndrico com $12 \mathrm{~cm}$ de altura e $10 \mathrm{~cm}$ de diâmetro. No topo da coluna ficava um módulo no qual era recolhida a espuma e despejada em um reservatório.

Através da Figura 1, pode-se observar o ponto de alimentação de água de lavagem (1) e coleta do concentrado (2), rotâmetro (3), bombas peristálticas (4,10 e 13), 5 e 6 indicam os pontos para amostragem do holdup, manômetros diferenciais (7), sistema de alimentação de ar comprimido com filtro de ar e rotâmetro em 8 e 9. É possível também identificar o Venturi (11) onde as bolhas são formadas, manômetro (12) e aerador (15). Na figura pode-se observar também o ponto de coleta do rejeito (15) e o sistema de amostragem de bolhas (16). 
Figura 1 - Unidade Experimental

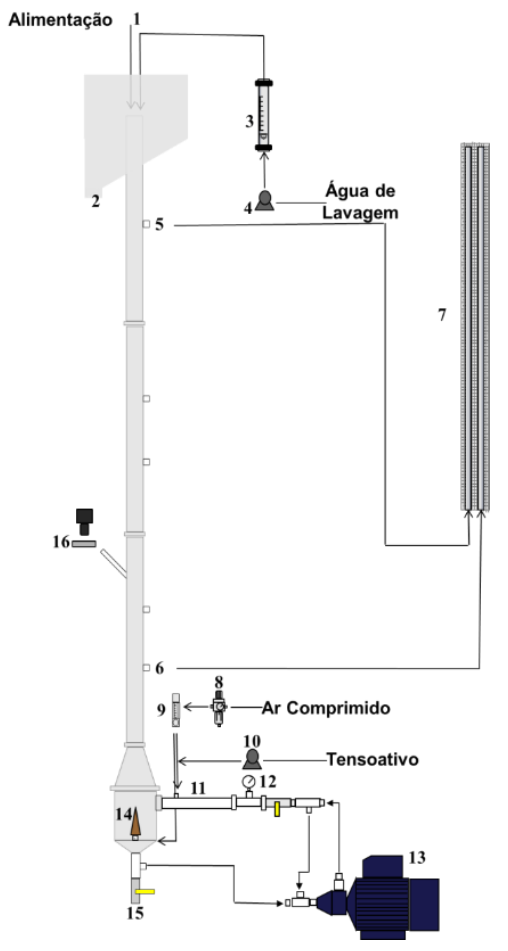

\subsection{Metodologias para a medida do diâmetro de bolha}

Método intrusivo: Neste método a amostragem de bolhas foi feita por meio da captura das bolhas no interior da coluna através de um amostrador. Desta forma, conforme mostrado na Figura 2, a coleta das bolhas foi realizada através de um tubo de aço, conectado ao amostrador, com $6 \mathrm{~cm}$ de diâmetro e $10 \mathrm{~cm}$ de comprimento posicionado no centro da coluna e com um ângulo de $45^{\circ}$ em relação à mesma. Com o auxílio de uma bomba peristáltica, posicionada à jusante do amostrador, as bolhas eram succionadas com velocidade previamente controlada de forma a diminuir o efeito de quebra e coalescência das bolhas coletadas.

Método não-intrusivo: Neste método a filmagem realizada obtinha imagem das bolhas em estado dinâmico, ou seja, as bolhas em movimento na coluna eram filmadas, com a mesma câmera de alta velocidade utilizada no método anterior, posicionada em paralelo com a coluna, como mostra a Figura 3. As filmagens foram realizadas a 250 quadros por segundo e reproduzidas com uma velocidade de 5 até 250 quadros por segundo para serem gravadas. $\mathrm{Na}$ seção onde a filmagem foi feita havia a presença de um cubo ao redor do cilindro o qual era preenchido com água durante as filmagens para corrigir a deformação causada nas bolhas pelo formato cilíndrico da coluna. 
Figura 2 - Esquema representativo dos métodos (a) - intrusivo e (b) - não-intrusivo

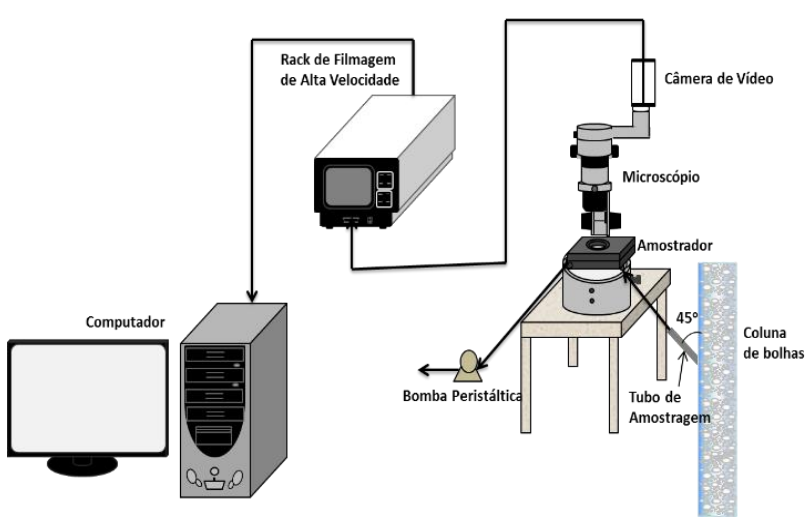

(a)
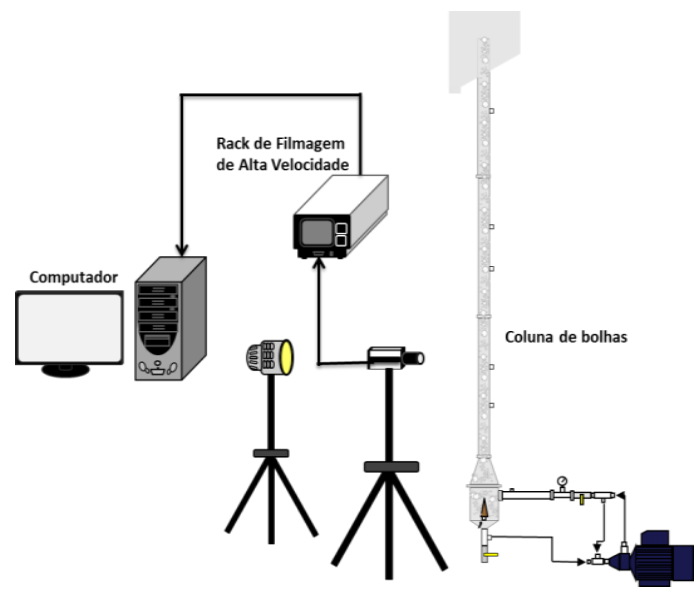

(b)

\subsection{Análise do Tamanho das Bolhas}

As filmagens obtidas por ambos os métodos foram divididas em frames, por meio do software ImageGrab, que gerou fotos para serem analisadas no software ImageJ, onde, os diâmetros das bolhas foram determinados pelo cálculo das áreas, a partir de uma calibração prévia com um padrão de comprimento conhecido. Nesse segundo software, com um padrão de comprimento conhecido era realizada a conversão da medida encontrada de pixels para milímetros. Sendo que para a obtenção da distribuição de diâmetro de bolhas foram medidas em média 500 bolhas para cada condição.

\subsection{Reagentes de flotação}

Os reagentes utilizados na flotação exercem uma função específica através da manipulação da química da polpa, pois promovem uma modificação seletiva na superfície dos minerais, de modo a afetar e induzir as propriedades de hidrofobicidade e hidrofilicidade e possibilitar a seletividade na separação.

Assim, foram utilizados nos testes de flotação como coletor o sabão de óleo de arroz (2,5\%), como depressor o fubá de milho gelatinizado $(3,0 \%)$ e para regulação do $\mathrm{pH}$ foi utilizada solução de hidróxido de sódio a $10 \%$. As dosagens utilizadas em cada teste foram de $110 \mathrm{~g} /$ ton de coletor e $700 \mathrm{~g} /$ ton de depressor. $\mathrm{O}$ valor do $\mathrm{pH}$ no condicionamento do minério foi mantido em 12 e a porcentagem de sólidos de $60 \%$, para a alimentação a porcentagem de sólidos utilizada foi de $16 \%$ e a pressão de operação na linha do Venturi era de 1 bar. 


\section{RESULTADOS E DISCUSSÃO}

As informações sobre as condições para a obtenção dos diferentes tamanhos de bolha no sistema ar-água, utilizando-se um aerador do tipo Venturi podem ser encontradas na Tabela 1. Foram avaliados os parâmetros vazão de $\operatorname{ar}\left(\mathrm{Q}_{\mathrm{ar}}\right)$, concentração de tensoativo $\left(\mathrm{C}_{\mathrm{t}}\right)$ e vazão da alimentação de tensoativo $\left(\mathrm{Q}_{\mathrm{t}}\right)$ para a geração de bolhas de diferentes tamanhos, sendo estas classificadas em: microbolhas ou bolhas pequenas $(<100 \mu \mathrm{m})(\mathrm{BP})$, bolhas intermediárias $(>100 \mu \mathrm{m}$ e $<1000 \mu \mathrm{m})(\mathrm{BI})$ e bolhas grandes $(>1000 \mu \mathrm{m})(\mathrm{BG})$.

Tabela 1 - Condições para obtenção dos diferentes tamanhos de bolha no sistema ar-água

\begin{tabular}{|c|c|c|c|c|c|c|c|c|}
\hline Teste & $\begin{array}{c}\mathrm{Q}_{\mathrm{ar}} \\
(\mathrm{L} / \mathrm{h})\end{array}$ & $\begin{array}{c}\mathrm{C}_{\mathrm{t}} \\
\mathrm{ppm}\end{array}$ & $\begin{array}{c}\mathrm{Q}_{\mathrm{t}} \\
(\mathrm{ml} / \mathrm{min})\end{array}$ & $\begin{array}{c}\mathrm{D}_{32} \\
(\mu \mathrm{m})\end{array}$ & $\begin{array}{c}\mathrm{D}_{\mathrm{m}} \\
(\mu \mathrm{m})\end{array}$ & $\% \mathrm{BP}$ & $\% \mathrm{BI}$ & $\% \mathrm{BG}$ \\
\hline 1 & 5 & 70 & 200 & 242 & 148 & 24 & 76 & 0 \\
\hline 2 & 5 & 50 & 200 & 270 & 177 & 14 & 86 & 0 \\
\hline 3 & 12,5 & 15 & 100 & 945 & 413 & 17 & 72 & 11 \\
\hline 4 & 40 & 15 & 200 & 1582 & 763 & 8 & 57 & 35 \\
\hline 5 & 80 & 40 & 50 & 2290 & 2161 & 0 & 0 & 100 \\
\hline 6 & 100 & 10 & 50 & 2886 & 2543 & 0 & 0 & 100 \\
\hline 7 & 100 & 15 & 50 & 2818 & 2523 & 0 & 0 & 100 \\
\hline
\end{tabular}

Analisando os resultados observa-se que as maiores porcentagens de bolhas grandes foram alcançadas nos testes 5,6 e 7, onde utilizou-se alta vazão de ar e valores menores para concentração e vazão de tensoativo. Nota-se também que nestes testes, o valor do diâmetro de Sauter $\left(D_{32}\right)$ e o diâmetro médio $\left(D_{m}\right)$ foram maiores que $1000 \mu \mathrm{m}$ (bolhas grandes). Já as bolhas pequenas foram obtidas, em maior proporção, nos testes 1 e 2 , nos quais foram usados altos valores para concentração e vazão de alimentação do tensoativo e baixos valores para vazão de ar. Nota-se que nestes testes, embora a porcentagem de bolhas pequenas não seja a maior, os valores de $\mathrm{D}_{32}$ e $\mathrm{D}_{\mathrm{m}}$ mostram uma distribuição na qual bolhas menores são predominantes e que não chegam a ser em sua maioria microbolhas, mas são bolhas pequenas $(<300 \mu \mathrm{m})$. Já nos testes 3 e 4 observa-se um valor de $D_{32}$ e $D_{m}$ maior que 100 e menor que $1000 \mu \mathrm{m}$ caracterizando forte presença de bolhas intermediárias o que pode ser comprovado pela porcentagem bem maior de bolhas intermediárias formadas.

O incremento da vazão de ar promove um regime turbulento na coluna, aumentando o contato entre as bolhas e a probabilidade de coalescência das mesmas, favorecendo, portanto, a obtenção de bolhas grandes (Reis e Barrozo, 2016), como se confirma baseado no $D_{m}$ e $D_{32}$ dos testes 5,6 e 7. Já o tensoativo, tem por função diminuir o efeito de coalescência (Reis e Barrozo, 2016), portanto, altas concentrações e vazões de tensoativo beneficiam a formação de bolhas menores, conforme se verifica com a análise do $\mathrm{D}_{32}$ e $\mathrm{D}_{\mathrm{m}}$ dos testes 1 e 2 .

Visto que no processo de flotação as bolhas de ar são responsáveis pela coleta e transporte das partículas hidrofóbicas da polpa para a espuma, torna-se de fundamental importância a determinação das condições ideais para a formação de diferentes tamanhos de bolha, de modo a garantir melhor eficiência no processo de flotação (Costa, 2006). Porém, a adição de partículas (minério) ao sistema causa muita influência no tamanho das bolhas previamente definidos, conforme pode ser observado na Tabela 2. 
Tabela 2 - Influência da adição do minério sobre o tamanho das bolhas

\begin{tabular}{|c|c|c|c|c|c|c|}
\hline Teste & $\begin{array}{c}\mathrm{D}_{32}(\mu \mathrm{m}) \\
\text { ar-água }\end{array}$ & $\begin{array}{c}\mathrm{D}_{\mathrm{m}}(\mu \mathrm{m}) \\
\text { ar-água }\end{array}$ & $\begin{array}{c}\mathrm{D}_{32}(\mu \mathrm{m}) \\
\text { minério }\end{array}$ & $\begin{array}{c}\mathrm{D}_{\mathrm{m}}(\mu \mathrm{m}) \\
\text { minério }\end{array}$ & $\begin{array}{c}\text { Redução } \\
\mathrm{D}_{32}\end{array}$ & $\begin{array}{c}\text { Redução } \\
\mathrm{D}_{\text {médio }}\end{array}$ \\
\hline 1 & 242 & 148 & 195 & 110 & $-19 \%$ & $-26 \%$ \\
\hline 2 & 270 & 177 & 236 & 141 & $-13 \%$ & $-20 \%$ \\
\hline 3 & 945 & 413 & 349 & 213 & $-63 \%$ & $-48 \%$ \\
\hline 4 & 1582 & 763 & 391 & 294 & $-75 \%$ & $-61 \%$ \\
\hline 5 & 2290 & 2161 & 997 & 777 & $-56 \%$ & $-64 \%$ \\
\hline 6 & 2886 & 2543 & 1302 & 1081 & $-55 \%$ & $-57 \%$ \\
\hline 7 & 2818 & 2523 & 1286 & 895 & $-64 \%$ & $-65 \%$ \\
\hline
\end{tabular}

Conforme pode-se observar nas duas últimas colunas da Tabela 2, ao se adicionar o minério à coluna de flotação, o tamanho das bolhas sofre um decréscimo significativo em relação aos dados obtidos no sistema ar-água. Tal efeito ocorre porque a adição de partículas ao sistema de flotação diminui a interação entre as bolhas, consequentemente diminuindo a coalescência entre as mesmas. Esse mesmo efeito foi observado por Reis e Barrozo (2016) utilizando sondas para a medida de tamanho de bolha durante a flotação. Assim esses resultados mostram que a técnica utilizada é eficiente para prever o comportamento das bolhas durante a flotação mineral.

É possível verificar também que a redução no tamanho das bolhas é mais notável nos testes onde a coalescência entre as bolhas era favorecida, ou seja, nos testes com altas vazões de ar e baixas concentrações e vazões de tensoativo (testes que favoreciam a formação de bolhas intermediárias e grandes).

\section{CONCLUSÃO}

Verificou-se que aeradores do tipo Venturi associados ao ajuste de parâmetros como: vazão de ar e concentração e vazão de tensoativo são capazes de gerar bolhas de diversos tamanhos. Observou-se também que a adição de partículas ao sistema de flotação causa uma diminuição no tamanho de bolhas em relação aos resultados obtidos no sistema ar-água, sendo esse efeito prejudicial a eficiência da flotação, visto que para cada tamanho de partícula no processo de flotação é indicado certos tamanhos de bolhas.

\section{REFERÊNCIAS BIBLIOGRÁFICAS}

COSTA, S. A. Estudos de processos de tratamento de água de produção. Monografia, Universidade Federal de Pernambuco, Recife, PE, 2006.

FILHO, A. M. R.; e CARVALHO G. R. L.; LUZ P. H. M.; REIS A. S.; BARROZO M. A. S. Influência da adição de tensoativo no tamanho de bolhas e holdup de ar na flotação em coluna. Congresso Brasileiro de Engenharia Química em Iniciação Científica, 2015.

FINCH, J. A.; NESSET, Jan E.; ACUÑA, Claudio. Role of frother on bubble production and behaviour in flotation. M. Engineering, v. 21, n. 12, p. 949-957, 2008.

REIS, A. S.; BARROZO, M. A. S. A study on bubble formation and its relation with the performance of apatite flotation. Elsevier. v 161. p 112-120. Faculty of Chemical Engineering, Federal University of Uberlândia, Uberlândia, Brazil, 2016. 